\title{
Z-Score Functions of Hesitant Fuzzy Sets
}

\author{
Zahari Md Rodzi $^{1,2,}$, Abd Ghafur Ahmad ${ }^{2}$, Norul Fadhilah Ismail ${ }^{3}$, Nur Lina Abdullah ${ }^{1}$ \\ ${ }^{1}$ Faculty of Computer and Mathematical Science, UiTM Cawangan Negeri Sembilan, Kampus Seremban, 70300 Seremban, Negeri \\ Sembilan, Malaysia \\ ${ }^{2}$ School of Mathematical Science, Faculty of Science and Technology, Universiti Kebangsaan Malaysia, 43600 UKM, Bangi, Selangor, \\ Malaysia \\ ${ }^{3}$ Faculty of Computer and Mathematical Science, UiTM Cawangan Negeri Sembilan, Kampus Kuala Pilah, 72000 Seremban, Negeri \\ Sembilan, Malaysia
}

Received February 4, 2021; Revised May 27, 2021; Accepted June 5, 2021

\section{Cite This Paper in the following Citation Styles}

(a): [1] Zahari Md Rodzi, Abd Ghafur Ahmad, Norul Fadhilah Ismail, Nur Lina Abdullah, "Z-Score Functions of Hesitant Fuzzy Sets," Mathematics and Statistics, Vol. 9, No. 4, pp. 445 - 455, 2021. DOI: 10.13189/ms.2021.090405.

(b): Zahari Md Rodzi, Abd Ghafur Ahmad, Norul Fadhilah Ismail, Nur Lina Abdullah (2021). Z-Score Functions of Hesitant Fuzzy Sets. Mathematics and Statistics, 9(4), 445 - 455. DOI: 10.13189/ms.2021.090405.

Copyright@2021 by authors, all rights reserved. Authors agree that this article remains permanently open access under the terms of the Creative Commons Attribution License 4.0 International License

\begin{abstract}
The hesitant fuzzy set (HFS) concept as an extension of fuzzy set (FS) in which the membership degree of a given element, called the hesitant fuzzy element (HFE), is defined as a set of possible values. A large number of studies are concentrating on HFE and HFS measurements. It is not just because of their crucial importance in theoretical studies, but also because they are required for almost any application field. The score function of HFE is a useful method for converting data into a single value. Moreover, the scoring function provides a much easier way to determine each alternative's ranking order for multi-criteria decision-making (MCDM). This study introduces a new hesitant degree of HFE and the z-score function of HFE, which consists of z-arithmetic mean, z-geometric mean, and z-harmonic mean. The z-score function is developed with four main bases: a hesitant degree of HFE, deviation value of HFE, the importance of the hesitant degree of HFE, $\alpha$, and importance of the deviation value of HFE, $\beta$. These three proposed scores are compared with the existing scores functions to identify the proposed z-score function's flexibility. An algorithm based on the z-score function was developed to create an algorithm solution to MCDM. Example of secondary data on supplier selection for automated companies is used to prove the algorithms' capability in ranking order for MCDM.
\end{abstract}

Keywords Hesitant Degree, Z-Arithmetic Mean, Z-Geometric Mean, Z-Harmonic Mean, HFS, Score Function, MCDM

\section{Introduction}

In several fields, the fuzzy set (FS) [1] theory is commonly and successfully used to model some kinds of uncertainty. Still, due to various causes of vagueness that exist in the idea, the downside of this method is that it will be much tougher to cope with imprecise and uncertain information.

Researchers recognized that FS theory has many disadvantages, but several enhancements have been done to improve the method's usage in real life applications. Some of the most known extensions of FSs include intuitionistic fuzzy sets (IFS) [2], interval-valued intuition fuzzy set (IVIFS) [3], fuzzy multiset [4] and fuzzy soft sets [5]. The hesitant fuzzy set (HFS) was initially implemented by Torra [6], [7]. They expanded FS to HFS since they discovered that an element's membership to a set is difficult to decide under a group setting due to doubts between a few different values. More and more scholars have been attracted to do research on HFS [8][17] and answer numerous decision-making issues in MCDM [18]-[24].

The measurements for HFEs and HFSs are the subject of an increasing number of studies. This is not only due to their basic significance in scientific research, but also because they are invaluable in nearly all fields of use [25]. $\mathrm{Xia}$ and $\mathrm{Xu}$ [26] defined a widely used score function which, by averaging the membership values in HFEs, the function can help to draw the required comparison. Liao and $\mathrm{Xu}$ [27] developed a two-step algorithmic technique to rank HFEs. The Score value-Variance was developed by 
considering Xia's score function and the variance value of the memberships. Zhang [28] developed a sort of score function that is a generalization of the score function of Xia by taking into account the variance of HFE memberships. A new enhanced score feature with role weighting details was proposed by Farhadinia [29]. More recently, Farhadinia [30] presented a collection of HFE score functions and defined the scheme for selecting a suitable score function from a set of score functions. A score function integrated with $\delta(0<\delta \leq 1)$ presented by Zhang and $\mathrm{Xu}$ [28] is a parameter defined by the decision makers that can be adjusted according to the reality of the situation.

Even though various types of excellent score functions have been generated in the sense of HFE, certain critical problems still need to be discussed. What if the HFEs cannot be distinguished by these score functions of various lengths and deviation? What if the various HFEs have the same score? The goal of this study is to suggest a score function incorporated with HFE's hesitant degree and deviation value that is both flexible and efficient and addresses certain limitations of the current score functions.

The presentation of this article is as follows. In section 2 , we recall some basic concepts of HFSs and existing score functions. In section 3, we propose the z-score function of the HFEs which consists of z-arithmetic mean, z-geometric mean and $\mathrm{z}$-harmonic mean. In section 4 , the weighted z-score algorithm used in MCDM is presented and examples of automotive industry supplier selection are provided to show the usability of the suggested algorithm. Finally, in section 5, we give the conclusion of this study.

\section{Preliminaries}

This section is dedicated to explaining the definitions relating to HFS, existing HFE score function, hesitant degree of HFE and deviation value of HFE.

Definition 1 [7] Let a set $X$ be fixed. Then a hesitant fuzzy set (HFS) $H$ on $X$ in terms of a function $h$ is that when applied to $X$, returns a subset of $[0,1]$. To be easily understood, $\mathrm{Xu}$ and Xia expressed the hesitant fuzzy set by

$$
H(x)=\left\{\left(x, h_{A}(x)\right): x \in X\right\}
$$

where $h_{A}(x)$ is a set of some different values in $[0,1]$ that is called hesitant fuzzy elements (HFEs), representing the possible membership degrees of the element $x \in X$ to $A$.

Definition 2 [26] Let $h=\left\{h_{1}, h_{2}, \ldots, h_{n}\right\}$ be an HFE. The following functions can be considered as the score index for HFEs:

$$
S(\tilde{h})=\frac{1}{n} \sum_{1=i}^{n}\left(h_{i}\right)
$$

where $n$ is the number of membership in $\tilde{h}$. For two HFEs, $\tilde{h}_{1}$ and $\tilde{h}_{2}$, if $S\left(\tilde{h}_{1}\right)>S\left(\tilde{h}_{2}\right)$ then $\tilde{h}_{1}$ is superior to $\tilde{h}_{2}$, denoted by $\tilde{h}_{1} \succ \tilde{h}_{2}$ and if $S\left(\tilde{h}_{1}\right)=S\left(\tilde{h}_{2}\right)$, then $\tilde{h}_{1}$ is indifferent from $\tilde{h}_{2}$ denoted by $\tilde{h}_{1} \sim \tilde{h}_{2}$.

Definition 3 [29] Let $h=\left\{h_{1}, h_{2}, \ldots, h_{n}\right\}$ be an HFE. The following functions can be considered as the score index for HFEs:

$$
S(\tilde{h})=\frac{\sum_{1}^{n} \beta(l) h_{i}}{\sum_{1}^{n} \beta(l)}
$$

where $\beta(l)(l=1,2,3, \ldots, n)$ is the order of monotonic increase of the positive value of the index $l$. For two HFEs, $\tilde{h}_{1}$ and $\tilde{h}_{2}$, if $S\left(\tilde{h}_{1}\right)>S\left(\tilde{h}_{2}\right)$ then $\tilde{h}_{1}$ is higher than $\tilde{h}_{2}$ represented by $\tilde{h}_{1} \succ \tilde{h}_{2}$ and if $S\left(\tilde{h}_{1}\right)=S\left(\tilde{h}_{2}\right)$, then $\tilde{h}_{1}$ is no difference to $\tilde{h}_{2}$ represented by $\tilde{h}_{1} \sim \tilde{h}_{2}$.

Definition 4 [27] Let $h=\left\{h_{1}, h_{2}, \ldots, h_{n}\right\}$ be an HFE. The following functions can be considered as the score index for HFEs:

$$
V(\tilde{h})=\frac{1}{n}\left(\sqrt{\sum_{i=1}^{n}\left(h_{i}-h_{n}\right)}\right)^{2}
$$

where $n$ is the number of elements in $\tilde{h}$ and $V(\tilde{h})$ that reflects the value of the variance between all possible values $\tilde{h}$. Based on $S(\tilde{h})$ and $V(\tilde{h})$, they introduced a score-variance model to give a ranking for HFEs.

Definition 5 [27] Let $\tilde{h}_{1}$ and $\tilde{h}_{2}$ be two HFEs, $S\left(\tilde{h}_{1}\right)$ and $S\left(\tilde{h}_{2}\right)$ is the score for $\tilde{h}_{1}$ and $\tilde{h}_{2}$ respectively. While, $V\left(\tilde{h}_{1}\right)$ and $V\left(\tilde{h}_{2}\right)$ are deviation value for $\tilde{h}_{1}$ and $\tilde{h}_{2}$, respectively. Then

1. If $S\left(\tilde{h}_{1}\right)>S\left(\tilde{h}_{2}\right)$, then $\tilde{h}_{1} \succ \tilde{h}_{2}$.

2. If $S\left(\tilde{h}_{1}\right)=S\left(\tilde{h}_{2}\right)$ then,

$$
\begin{array}{cl}
\text { i. } & \text { If } V\left(\tilde{h}_{1}\right)>V\left(\tilde{h}_{2}\right) \text {, then } \tilde{h}_{1} \prec \tilde{h}_{2} . \\
\text { ii. } & \text { If } V\left(\tilde{h}_{1}\right)<V\left(\tilde{h}_{2}\right) \text {, then } \tilde{h}_{1} \succ \tilde{h}_{2} . \\
\text { iii. } & \text { If } V\left(\tilde{h}_{1}\right)=V\left(\tilde{h}_{2}\right) \text {, then } \tilde{h}_{1}=\tilde{h}_{2} .
\end{array}
$$

Definition 6 [30] Let $h=\left\{h_{1}, h_{2}, \ldots, h_{n}\right\}$ be an HFE. The following functions can be considered as the score index for HFEs:

1. The arithmetic-mean score function

$$
S_{A M}(\tilde{h})=\frac{1}{n} \sum_{1=i}^{n} h_{i}
$$

2. The geometric-mean score function:

$$
S_{G M}(\tilde{h})=\left(\prod_{1=i}^{n}\left(h_{i}\right)\right)^{\frac{1}{n}}
$$

3. The minimum score function:

$$
S_{\min }(\tilde{h})=\min \left\{\left(h_{1}\right),\left(h_{2}\right), \ldots,\left(h_{n}\right)\right\} .
$$


4. The maximum score function:

$$
S_{\max }(\tilde{h})=\max \left\{\left(h_{1}\right),\left(h_{2}\right), \ldots,\left(h_{n}\right)\right\} .
$$

5. The product score function: $S_{P}(\tilde{h})=\prod_{1=i}^{n} h_{i}$

6. The fractional score function:

$$
S_{F}(\tilde{h})=\frac{\prod_{1=i}^{n} h_{i}}{\prod_{1=i}^{n} h_{i}+\prod_{1=i}^{n}\left(1-h_{i}\right)}
$$

Definition 7 [28] Let $h=\left\{h_{1}, h_{2}, \ldots, h_{n}\right\}$ be an HFE. The following function can be considered as the score index for HFE: $S(\tilde{h})=\left(\frac{h_{1}^{\delta}+h_{2}^{\delta}+\ldots+h_{n}^{\delta}}{n}\right)^{\frac{1}{\delta}}$

where $\delta(0<\delta \leq 1)$ is a parameter to be determined by the decision makers where $n$ is the number of elements in $\tilde{h}$. Let $\tilde{h}_{1}$ and $\tilde{h}_{2}$ be two HFEs, if $S\left(\tilde{h}_{1}\right)>S\left(\tilde{h}_{2}\right)$ then $\tilde{h}_{1}$ is superior to $\tilde{h}_{2}$ denoted by $\tilde{h}_{1} \succ \tilde{h}_{2}$ and if $S\left(\tilde{h}_{1}\right)=S\left(\tilde{h}_{2}\right)$ then $\tilde{h}_{1}$ is indifferent to $\tilde{h}_{2}$ denoted by $\tilde{h}_{1} \sim \tilde{h}_{2}$.

Definition 8 [31] Let $\tilde{h}=\left\{h_{1}, h_{2}, \ldots, h_{n}\right\}$ is an HFE and $n$ be the length of $\tilde{h}$. The hesitant degree of HFE, $H_{d}(\tilde{h})$ is denoted by,

$$
H_{d}(\tilde{h})=1-\frac{1}{n} .
$$

The greater value of $H_{d}(\tilde{h})$, the more unconvinced the decision-maker about assessing an object or criterion. For example, if $n=1$, then $H(\tilde{h})=0$, this indicates that decision makers are able to determine the exact value of membership with confidence. This means the decision makers have no hesitance to determine the value of membership. However, $H(\tilde{h}) \approx 1$ indicates that the decision maker is fully skeptical, and almost unable to determine the value of the membership.

Definition 9 [32] Let $\tilde{h}=\left\{h_{1}, h_{2}, \ldots, h_{n}\right\}$ is an HFE and $n$ be the length of $\tilde{h}$. The deviations value of HFEs, $V_{Z}(\tilde{h})$ is denoted by

$$
V_{Z}(\tilde{h})=\sqrt{\sum_{1=i}^{n} \frac{\left(h_{i}-s(\tilde{h})\right)^{2}}{n}}
$$

where $s(\tilde{h})$ is the score of HFE.

\section{Z-Score Functions of HFEs}

The score function for the HFE plays an important role in determining the single value to represent the elements. The z-score function of the HFEs consists of z-arithmetic mean, z-geometric mean and z-harmonic mean. The suggested z-score functions take into account four fundamental aspects;

\section{Hesitant degree of HFEs}

The varying hesitant degree of HFE depends on the number of memberships for the element. The greater the membership of an element, the greater the value of the hesitant degree of HFE.

\section{Deviation value of HFEs}

This value measures the total variation or spread of a group of membership in elements. Low deviation value indicates that membership tends to approach the mean from the set, while high deviation value indicates that its membership spreads across a wider range.

3. Importance of the hesitant degree of HFE, $\alpha$

Parameter $\alpha \in[0,1]$ explains the importance of the hesitant degree of HFEs in determining the z-arithmetic mean, z-geometric mean, and z-harmonic mean score values. The higher the parameter, $\alpha$, the more important the hesitant degree of HFE in determining the z-score.

4. Importance of the deviation value of HFE, $\beta$

This value explains the importance of the deviation value of HFE in determining the $z$-arithmetic mean, z-geometric mean, and z-harmonic mean score values. The parameter $\beta \in[0,1]$ is the value used by the decision maker to determine the $\mathrm{z}$-score-z value. The higher the value of $\beta$ means the more important the deviation value of HFE in determining the z-score.

Definition 10 Let $\tilde{h}=\left\{h_{1}, h_{2}, \ldots, h_{n}\right\}$ is an HFE and $n$ is the length of $\tilde{h}$. The hesitant degree of HFEs, $H_{\mathrm{dz}}(\tilde{h})$ is denoted by:

$$
H_{\mathrm{d} Z}(\tilde{h})=\frac{n-1}{\chi}
$$

where $\chi$ is determined by $n$. If $n \leq 10$, then $\chi=10$, and if $n>10$ then $\chi=n$. The greater the value of $H_{\mathrm{dZ}}(\tilde{h})$, the more unconvinced the decision maker in assessing an object or criterion. We believe that each unit increase in membership of element (length of $\tilde{h}$ ) is equivalent to the increase in the hesitant degree of HFEs.

Example 3.2. Let $\tilde{h}_{1}=\{0.2,0.4,0.6\}$ be an HFE. Then, the hesitant degree for $\tilde{h}_{1}$ is given by

$$
\begin{aligned}
H_{\mathrm{dZ}}\left(\tilde{h}_{1}\right) & =\frac{n-1}{\chi} \\
& =\frac{3-1}{10} \\
& =0.2
\end{aligned}
$$




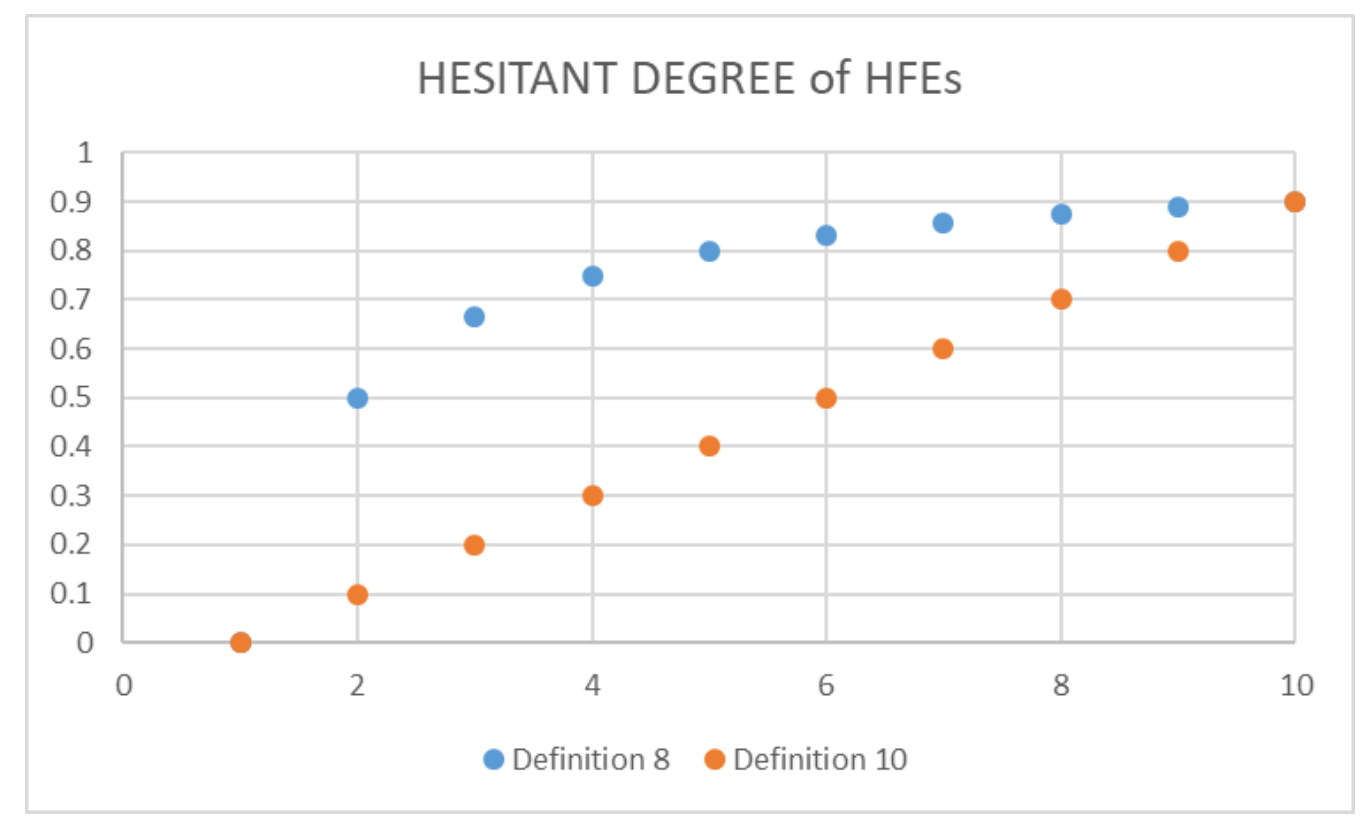

Figure 1. Value of hesitant degree of HFEs

The value of the hesitant degree of HFE depends on the number of membership in HFE. The larger the number of membership means more sceptical the decision makers are about assessing an obiect or criterion. For examble. when the length of $\tilde{h}$ is 1 , then the hesitant degree of HFE is 0 which means that the decision makers are not hesitant in assessing an object or criterion. Based on Definition 8, decision makers tend to choose smaller numbers of membership due to significant differences in each membership addition, for example, if the number of elements is two or more, the hesitant value will be equivalent to 0.5 or greater. This will result in the purpose of HFS to be eroded.

Definition 11 Let $\tilde{h}=\left\{h_{1}, h_{2}, \ldots, h_{n}\right\}$ be an HFE, z-arithmetic mean score function for the HFE is given as:

$$
S z_{A M}(\tilde{h})=\frac{1}{n} \sum_{1=i}^{n}\left(h_{i}\right) \cdot\left(1-H_{d Z}(\tilde{h})\right)^{\alpha} \cdot\left(1-V_{Z}(\tilde{h})\right)^{\beta}
$$

Definition 12 Let $\tilde{h}=\left\{h_{1}, h_{2}, \ldots, h_{n}\right\}$ be an HFE, z-geometric mean score function for the HFE is given as:

$$
S z_{G M}(\tilde{h})=\left(\prod_{1=i}^{n}\left(h_{i}\right)\right)^{\frac{1}{n}} \cdot\left(1-H_{d Z}(\tilde{h})\right)^{\alpha} \cdot\left(1-V_{Z}(\tilde{h})\right)^{\beta}
$$

Definition 13 Let $\tilde{h}=\left\{h_{1}, h_{2}, \ldots, h_{n}\right\}$ be an HFE, z-harmonic mean score function for the HFE is given as:

$$
S Z_{H M}(\tilde{h})=\frac{n}{\frac{1}{h_{1}}+\frac{1}{h_{2}}+\ldots+\frac{1}{h_{n}}} \cdot\left(1-H_{d Z}(\tilde{h})\right)^{\alpha} \cdot\left(1-V_{Z}(\tilde{h})\right)^{\beta}
$$

This suggests, for instance, that $(\alpha, \beta)=(0,0)$ considers a hesitant degree of HFE and deviation value of
HFE not to be significant in deciding HFE $\mathrm{z}$-score. If $(\alpha, \beta)=(1,1)$, this shows that decision makers feel that both hesitant degree of HFE and deviations value of HFE are indispensable for determining HFE z-score. If $(\alpha, \beta)=(0,1)$, this shows that decision makers feel the hesitant degree of HFE is not required while the deviation value of HFE is indispensable for determining the HFE z-score.

For a single membership HFEs, $\tilde{h}=\{h\}$, then $S z_{A M}(\tilde{h})=h, S z_{G M}(\tilde{h})=h$ and $S z_{H M}(\tilde{h})=h$. For two HFEs, $\tilde{h}_{1}$ and $\tilde{h}_{2}$, if $s\left(\tilde{h}_{1}\right)>s\left(\tilde{h}_{2}\right)$ the $\tilde{h}_{1}$ is superior to $\tilde{h}_{2}$ denoted by $\tilde{h}_{1} \succ \tilde{h}_{2}$ and if $s\left(\tilde{h}_{1}\right)=s\left(\tilde{h}_{2}\right)$, then $\tilde{h}_{1}$ is indifferent to $\tilde{h}_{2}$ denoted by $\tilde{h}_{1} \sim \tilde{h}_{2}$.

Proposition 1. For any HFE, $\tilde{h}=\left\{h_{1}, h_{2}, \ldots, h_{n}\right\}$. The score functions $S z_{A M}(\tilde{h}), S z_{G M}(\tilde{h})$ dan $S z_{H M}(\tilde{h}) \in[0,1]$ show that the hesitant degree of HFE is given as $H_{\mathrm{dZ}}(\tilde{h})=\frac{n-1}{\chi} \leq 1$

Notice that,

$$
\chi \geq n \Rightarrow \frac{1}{\chi} \leq \frac{1}{n} \Rightarrow \frac{n-1}{\chi} \leq \frac{n-1}{n}=\frac{n}{n}-\frac{1}{n}=1-\frac{1}{n} \leq 1,
$$

then $0 \leq H_{d Z}(\tilde{h})=\frac{n-1}{\chi} \leq 1$.

Therefore, $\left.\quad 0 \leq 1-H_{\mathrm{dZ}}(\tilde{h})\right) \leq 1 \quad$ and next $0 \leq\left(1-H_{\mathrm{dZ}}(\tilde{h})\right)^{\alpha} \leq 1$ or $\left(1-H_{\mathrm{dZ}}(\tilde{h})\right)^{\alpha} \in[0,1]$.

The HFE deviation values are as follows, 


$$
\begin{aligned}
V_{Z}(\tilde{h}) & =\sqrt{\frac{\sum_{1=i}^{n}\left(h_{i}-\bar{h}\right)^{2}}{n}} \\
V_{Z}(\tilde{h})^{2} & =\frac{\sum_{1=i}^{n}\left(h_{i}-\bar{h}\right)^{2}}{n} \\
& =\frac{\sum_{1=i}^{n}\left(h_{i}^{2}-2 h_{i} \bar{h}-\bar{h}^{2}\right)}{n} \\
V_{Z}(\tilde{h})^{2} & =\frac{\sum_{1=i}^{n} h_{i}^{2}}{2 n}-\frac{2 \bar{h} \sum_{1=i}^{n} h_{i}}{n}-\frac{n \bar{h}^{2}}{n} \\
& =\frac{\sum_{1=i}^{n} h_{i}^{2}}{\sum_{1=i}^{n} h_{i}^{2}}-2 \bar{h}^{2}-\bar{h}^{2} \\
& =\frac{\bar{h}^{2}}{n}
\end{aligned}
$$

and then it can be proven that

$$
\begin{gathered}
\frac{\sum_{1=i}^{n} h_{i}^{2}}{n} \geq \bar{h}^{2}, \\
\sum_{i=1}^{n} h_{i}^{2}=\sum_{i \overline{\bar{n} 1}}^{n}\left(h_{i}-\bar{h}+\bar{h}\right)^{2} \\
=\sum_{i=1}^{i \bar{n} 1}\left(\left(h_{i}-\bar{h}\right)^{2}+2 \bar{h}\left(h_{i}-\bar{h}\right)+\bar{h}^{2}\right) \\
=\sum_{i=1}^{n}\left(h_{i}-\bar{h}\right)^{2}+2 \bar{h}\left(\sum_{i=1}^{n} h_{i}-n \bar{h}\right)+n \bar{h}^{2} \\
=n \bar{h}^{2}+\sum_{i=1}^{n}\left(h_{i}-\bar{h}\right)^{2} \\
\geq n \bar{h}^{2} \quad \\
\frac{\sum h_{i}^{2}}{n} \geq \bar{h}^{2}
\end{gathered}
$$

Therefore $0 \leq V_{Z}(\tilde{h})=\sqrt{\sum_{1=i}^{n} \frac{\left(h_{i}-\bar{h}\right)^{2}}{n}} \leq 1$, and this proves that $V_{Z}(\tilde{h}) \in[0,1]$.

Now, let $h^{+}=\max \left\{h_{1}, h_{2}, \ldots, h_{n}\right\}$ and $h^{-}=\min \left\{h_{1}, h_{2}, \ldots, h_{n}\right\}$ $h_{i} \in[0,1]$, then the arithmetic mean, geometric mean and harmonic mean are given as

$$
\begin{aligned}
& \frac{h_{1}+h_{2}+\ldots+h_{n}}{n} \leq \frac{h^{+}+h^{+}+\ldots+h^{+}}{n} \\
& =\frac{n h^{+}}{n}=h^{+} \leq 1,
\end{aligned}
$$

and

$$
\begin{aligned}
& \frac{h_{1}+h_{2}+\ldots+h_{n}}{n} \geq \frac{h^{-}+h^{-}+\ldots+h^{-}}{n} \\
& =\frac{n h^{-}}{n}=h^{-} \geq 0
\end{aligned}
$$

This proves that arithmetic mean is $\frac{1}{n} \sum_{1=i}^{n}\left(h_{i}\right) \in[0,1]$.

While, for the geometric mean, let,

$$
\begin{aligned}
& \left(h_{1} \times h_{2} \times \ldots \times h_{n}\right)^{\frac{1}{n}} \leq\left(h^{+} \times h^{+} \times \ldots \times h^{+}\right)^{\frac{1}{n}} \\
& \left(\left(h^{+}\right)^{n}\right)^{\frac{1}{n}}=h^{+} \leq 1,
\end{aligned}
$$

and

$$
\begin{aligned}
& \left(h_{1} \times h_{2} \times \ldots \times h_{n}\right)^{\frac{1}{n}} \leq\left(h^{-} \times h^{-} \times \ldots \times h^{-}\right)^{\frac{1}{n}} \\
& \left(\left(h^{-}\right)^{n}\right)^{\frac{1}{n}}=h^{0} \leq 0
\end{aligned}
$$

Then, the geometric mean is proved to be $\left(\prod_{1=i}^{n}\left(h_{i}\right)\right)^{\frac{1}{n}} \in[0,1]$. Meanwhile for harmonic mean,

$$
\begin{aligned}
& \frac{n}{\frac{1}{h_{1}}+\frac{1}{h_{2}}+\ldots+\frac{1}{h_{n}}} \leq \frac{n}{\frac{1}{h^{+}}+\frac{1}{h^{+}}+\ldots+\frac{1}{h^{+}}} \\
& =\frac{n}{n \cdot \frac{1}{h^{+}}}=\frac{n}{n} h^{+}=h^{+} \geq 1,
\end{aligned}
$$

and

$$
\begin{aligned}
& \frac{n}{\frac{1}{h_{1}}+\frac{1}{h_{2}}+\ldots+\frac{1}{h_{n}}} \leq \frac{n}{\frac{1}{h^{-}}+\frac{1}{h^{-}}+\ldots+\frac{1}{h^{-}}} \\
& =\frac{n}{n \cdot \frac{1}{h^{-}}}=\frac{n}{n} h^{-}=h^{-} \leq 0
\end{aligned}
$$

Then the harmonic mean is proved to be $\frac{n}{\frac{1}{h_{1}}+\frac{1}{h_{2}}+\ldots+\frac{1}{h_{n}}} \in[0,1]$.

Next $h_{i}=[0,1], \alpha=[0,1]$ and $\beta=[0,1]$.

and

$S z_{A M}(\tilde{h})=\underbrace{\left(\frac{1}{n} \sum_{1=i}^{n}\left(h_{i}\right) \cdot\right)}_{\in[0,1]} \underbrace{\left(1-H_{d Z}(\tilde{h})\right)^{\alpha}}_{\in[0,1]} \cdot \underbrace{\left(1-V_{Z}(\tilde{h})\right)^{\beta}}_{\in[0,1]} \leq 1$. so

$S z_{A M}(\tilde{h}) \in[0,1]$

It is also proven for $S z_{G M}(\tilde{h}) \in[0,1]$ and $S z_{H M}(\tilde{h}) \in[0,1]$.

Proposition 2. For a single-valued HFE $\tilde{h}=\{h\}$, the proposed z-score functions: $S z_{A M}(\tilde{h})=h, S z_{G M}(\tilde{h})=h$ dan $S z_{H M}(\tilde{h})=h$. In particular, if $\tilde{h}$ is the hesitant empty element $\tilde{h}=\{0\}$, then $S z_{A M}(\tilde{h})=0, S z_{G M}(\tilde{h})=0$ 
and $S z_{H M}(\tilde{h})=0$. If $\tilde{h}$ is the hesitant full element, $\tilde{h}=\{1\}$ then, $S z_{A M}(\tilde{h})=1, S z_{G M}(\tilde{h})=1$ and $S z_{H M}(\tilde{h})=1$.

Proof. The proof is obvious.

Example 3.2 [28] Let $\tilde{h}_{1}=\{0.3,0.5\} \quad \tilde{h}_{2}=\{0.4\}$ and $\tilde{h}_{3}=\{0.2,0.4,0.6\}$ be three HFEs. The ranking of proposed z-score functions is given in Table 1 until Table 3.

The tables show that the ranking for the three HFEs are the same for each $(\alpha, \beta)$ with $\tilde{h}_{3} \prec \tilde{h}_{1} \prec \tilde{h}_{2}$ except for z-arithmetic mean with $(\alpha, \beta)=(0,0)$, where the ranking is $\tilde{h}_{1} \sim \tilde{h}_{2}-\tilde{h}_{3}$ and equals to Definition 2 .

Table 1. The score of z-arithmetic mean

\begin{tabular}{|c|c|c|c|c|c|}
\hline & $(\alpha, \beta)=(0,0)$ & $(\alpha, \beta)=(0,1)$ & $(\alpha, \beta)=(0.5,0.5)$ & $(\alpha, \beta)=(1,0)$ & $(\alpha, \beta)=(1,1)$ \\
\hline$\tilde{h}_{1}$ & 0.40 & 0.36 & 0.36 & 0.36 & 0.324 \\
\hline$\tilde{h}_{2}$ & 0.40 & 0.40 & 0.40 & 0.40 & 0.4 \\
\hline$\tilde{h}_{3}$ & 0.40 & 0.33 & 0.33 & 0.32 & 0.27 \\
\hline & $\tilde{h}_{1} \sim \tilde{h}_{2}-\tilde{h}_{3}$ & $\tilde{h}_{3} \prec \tilde{h}_{1} \prec \tilde{h}_{2}$ & $\tilde{h}_{3} \prec \tilde{h}_{1} \prec \tilde{h}_{2}$ & $\tilde{h}_{3} \prec \tilde{h}_{1} \prec \tilde{h}_{2}$ & $\tilde{h}_{3} \prec \tilde{h}_{1} \prec \tilde{h}_{2}$ \\
\hline
\end{tabular}

Table 2. The score of z-geometric mean

\begin{tabular}{|c|c|c|c|c|c|}
\hline & $(\alpha, \beta)=(0,0)$ & $(\alpha, \beta)=(0,1)$ & $(\alpha, \beta)=(0.5,0.5)$ & $(\alpha, \beta)=(1,0)$ & $(\alpha, \beta)=(1,1)$ \\
\hline$\tilde{h}_{1}$ & 0.3873 & 0.3486 & 0.3486 & 0.3486 & 0.3137 \\
\hline$\tilde{h}_{2}$ & 0.4000 & 0.4000 & 0.4000 & 0.4000 & 0.4000 \\
\hline$\tilde{h}_{3}$ & 0.3634 & 0.3041 & 0.2973 & 0.2907 & 0.2433 \\
\hline & $\tilde{h}_{3} \prec \tilde{h}_{1} \prec \tilde{h}_{2}$ & $\tilde{h}_{3} \prec \tilde{h}_{1} \prec \tilde{h}_{2}$ & $\tilde{h}_{3} \prec \tilde{h}_{1} \prec \tilde{h}_{2}$ & $\tilde{h}_{3} \prec \tilde{h}_{1} \prec \tilde{h}_{2}$ & $\tilde{h}_{3} \prec \tilde{h}_{1} \prec \tilde{h}_{2}$ \\
\hline
\end{tabular}

Table 3. The score of z-harmonic mean

\begin{tabular}{|c|c|c|c|c|c|}
\hline & $(\alpha, \beta)=(0,0)$ & $(\alpha, \beta)=(0,1)$ & $(\alpha, \beta)=(0.5,0.5)$ & $(\alpha, \beta)=(1,0)$ & $(\alpha, \beta)=(1,1)$ \\
\hline$\tilde{h}_{1}$ & 0.3750 & 0.3375 & 0.3375 & 0.3375 & 0.3038 \\
\hline$\tilde{h}_{2}$ & 0.4000 & 0.4000 & 0.4000 & 0.4000 & 0.4000 \\
\hline$\tilde{h}_{3}$ & 0.3273 & 0.2738 & 0.2678 & 0.2618 & 0.2191 \\
\hline & $\tilde{h}_{3} \prec \tilde{h}_{1} \prec \tilde{h}_{2}$ & $\tilde{h}_{3} \prec \tilde{h}_{1} \prec \tilde{h}_{2}$ & $\tilde{h}_{3} \prec \tilde{h}_{1} \prec \tilde{h}_{2}$ & $\tilde{h}_{3} \prec \tilde{h}_{1} \prec \tilde{h}_{2}$ & $\tilde{h}_{3} \prec \tilde{h}_{1} \prec \tilde{h}_{2}$ \\
\hline
\end{tabular}

Table 4. HFEs ranking based on score functions

\begin{tabular}{|c|c|}
\hline Score function & Ranking \\
\hline Xia and Xu (2011) & $\tilde{h}_{1} \sim \tilde{h}_{2}-\tilde{h}_{3}$ \\
\hline Farhadinia (2012) & $\tilde{h}_{1} \prec \tilde{h}_{2} \prec \tilde{h}_{3}$ \\
\hline Liao and Xu (2013) & $\tilde{h}_{3} \prec \tilde{h}_{1} \prec \tilde{h}_{2}$ \\
\hline Zhang and Xu (2014) & $\tilde{h}_{3} \prec \tilde{h}_{1} \prec \tilde{h}_{2}$ \\
\hline Farhadinia (Geometric) (2014) & $\tilde{h}_{3} \prec \tilde{h}_{1} \prec \tilde{h}_{2}$ \\
\hline Farhadinia (Minimum) (2014) & $\tilde{h}_{3} \prec \tilde{h}_{1} \prec \tilde{h}_{2}$ \\
\hline Farhadinia (Maximum) (2014) & $\tilde{h}_{3} \prec \tilde{h}_{1} \prec \tilde{h}_{2}$ \\
\hline Farhadinia (Product) (2014) & $\tilde{h}_{3} \prec \tilde{h}_{1} \prec \tilde{h}_{2}$ \\
\hline Farhadinia (Fraction) (2014) & $\tilde{h}_{3} \prec \tilde{h}_{1} \prec \tilde{h}_{2}$ \\
\hline Z-arithmetic mean $(\alpha, \beta)=(1,1)$ & $\tilde{h}_{3} \prec \tilde{h}_{1} \prec \tilde{h}_{2}$ \\
\hline Z-geometric mean $(\alpha, \beta)=(1,1)$ & $\tilde{h}_{3} \prec \tilde{h}_{1} \prec \tilde{h}_{2}$ \\
\hline Z-harmonic mean $(\alpha, \beta)=(1,1)$ & $\tilde{h}_{3} \prec \tilde{h}_{1} \prec \tilde{h}_{2}$ \\
\hline
\end{tabular}

The same examples introduced by $\mathrm{Xu}$ and Xia (2012) are used to analyse and compare the ranking with various methods. The comparison results are shown in Table 4.

$\mathrm{Xu}$ and Xia [26] approach only considers the mean value of all elements in HFE and does not distinguish between these three HFEs. This approach does not take into account the length of the HFEs. Meanwhile, the ranking based on Farhadinia's score approach [29] is inconsistent with others. Since the methods of Liao and $\mathrm{Xu}$ [27] are compatible with others, it takes time. The methodology is based on two principles, and if we need to address the issue in MCDM, it is very complicated to implement.

The score function of Zhang and $\mathrm{Xu}$ [28] is consistent with most of the existing score functions. However, the function of this score does not consider the spread of membership of HFEs. For the minimum and maximum score function, the ranking is consistent but does not consider the deviation value and length of HFEs. This score functions only consider the value of the first and last 
membership of each HFE.

Example 3.3. Let $\tilde{h}_{1}=\{0.1,0.4,0.9\}, \tilde{h}_{2}=\{0.4\}$ and $\tilde{h}_{3}=\{0.1,0.9\}$ be a three HFEs. The following comparisons are between the score functions of the z-arithmetic mean using the Zhang and $\mathrm{Xu}$ [28] approach. Table 5 is the score value and HFE ranking for both approaches.

Example 3.4 Let $\tilde{h}_{1}=\{0.1,0.3,0.8\}, \tilde{h}_{2}=\{0.1,0.7\}$, $\tilde{h}_{3}=\{0.2,0.6\}, \tilde{h}_{4}=\{0.3,0.5\}$ and $\tilde{h}_{5}=\{0.4\}$ be a five HFEs. The following comparisons are between score functions of z-arithmetic mean with Zhang and $\mathrm{Xu}$ [28] as per Table 6.

Based on Table 5 and Table 6, the ranking for Zhang and $\mathrm{Xu}$ approach [28] is the same for both examples given
Zhang and Xu used $\delta=0.5 \delta=1$, while the z-arithmetic mean is $(\alpha, \beta)=(0.5,0.5)$ and $(\alpha, \beta)=(1,1)$. This shows that Zhang and $\mathrm{Xu}$ [28] approach cannot distinguish HFE for both examples.

Example 3.5. Let $\tilde{h}_{1}=\{0.1,0.4,0.6\}$, $\tilde{h}_{2}=\{0.1,0.3,0.8\}$ and $\tilde{h}_{3}=\{0.2,0.3,0.4\}$, are three examples of HFE. Table 6 shows a comparison between the geometric mean score function and z-geometric mean.

Based on Table 7, the geometric score function is the same for all three HFEs, although the grading arrangement for the z-geometric mean is different that is $\tilde{h}_{3} \prec \tilde{h}_{2} \prec \tilde{h}_{1}$. The z-geometric mean takes into account the deviation degree and hesitant degree for these three HFEs.

Table 5. Comparison of a ranking order by Zhang and Xu (2014) and z-arithmetic mean

\begin{tabular}{|c|c|c|c|c|}
\hline Score function & $\tilde{h}_{1}$ & $\tilde{h}_{2}$ & $\tilde{h}_{3}$ & Ranking \\
\hline Zhang and Xu (2014) $\delta=0.5$ & 0.4 & 0.4 & 0.4 & $\tilde{h}_{1} \sim \tilde{h}_{2} \sim \tilde{h}_{3}$ \\
\hline Z-arithmetic mean $(\alpha, \beta)=(0.5,0.5)$ & 0.3417 & 0.4 & 0.3674 & $\tilde{h}_{3} \prec \tilde{h}_{1} \prec \tilde{h}_{2}$ \\
\hline
\end{tabular}

Table 6. Comparison of a ranking order by Zhang and Xu (2014) and z-arithmetic mean

\begin{tabular}{|c|c|c|c|c|c|c|}
\hline Score function & $\tilde{h}_{1}$ & $\tilde{h}_{2}$ & $\tilde{h}_{3}$ & $\tilde{h}_{4}$ & $\tilde{h}_{5}$ & Ranking \\
\hline Zhang and Xu (2014) $\delta=1$ & 0.4 & 0.4 & 0.4 & 0.4 & 0.4 & $\tilde{h}_{1} \sim \tilde{h}_{2} \sim \tilde{h}_{3} \sim \tilde{h}_{4} \sim \tilde{h}_{5}$ \\
\hline $\begin{array}{c}\text { Z-arithmetic mean } \\
(\alpha, \beta)=(1,1)\end{array}$ & 0.226 & 0.252 & 0.288 & 0.324 & 0.4 & $\tilde{h}_{5} \prec \tilde{h}_{4} \prec \tilde{h}_{3} \prec \tilde{h}_{2} \prec \tilde{h}_{1}$ \\
\hline
\end{tabular}

Table 7. Comparison of a ranking order by Farhadinia (2014) (geometric mean) with z-geometric mean

\begin{tabular}{|c|c|c|c|c|}
\hline Score function & $\tilde{h}_{1}$ & $\tilde{h}_{2}$ & $\tilde{h}_{3}$ & 0.2885 \\
\hline Geometric mean & 0.2885 & 0.2885 & 0.2860 & $\tilde{h}_{1} \sim \tilde{h}_{2} \sim \tilde{h}_{3}$ \\
\hline $\begin{array}{l}\text { Z-geometric mean } \\
(\alpha, \beta)=(0,0.1)\end{array}$ & 0.2836 & 0.2825 & $\tilde{h}_{3} \prec \tilde{h}_{2} \prec \tilde{h}_{1}$ \\
\hline
\end{tabular}




\section{Application of Z-Score Functions in MCDM}

The weighted z-score algorithm in MCDM is covered in this section, and examples of automotive industry supplier selection are provided to demonstrate the usability of the proposed algorithms.

Definition 3.12 Let $H=\left\{\tilde{h}_{1}, \tilde{h}_{2}, \ldots, \tilde{h}_{n}\right\}$ be an HFS. The function of the weighted z-arithmetic mean score for HFS is:

$$
S w_{A M}(H)=\sum_{i=1}^{k} w_{i} \cdot S z_{A M}(\tilde{h})
$$

Definition 3.13 Let $H=\left\{\tilde{h}_{1}, \tilde{h}_{2}, \ldots, \tilde{h}_{n}\right\}$ be an HFS. The function of the weighted z-geometric mean score for HFS is:

$$
S w_{G M}(H)=\sum_{i=1}^{k} w_{i} \cdot S z_{G M}(\tilde{h})
$$

Definition 3.14 Let $H=\left\{\tilde{h}_{1}, \tilde{h}_{2}, \ldots, \tilde{h}_{n}\right\}$ be an HFS. The function of the weighted z-harmonic mean score for HFS is:

$$
S w_{G M}(H)=\sum_{i=1}^{k} w_{i} \cdot S z_{H M}(\tilde{h})
$$

\section{Z-Score Algorithm in MCDM;}

Step 1. Build a decision matrix of HFS.

Step 2. Identify benefit criteria and cost criteria. If there is a cost criterion change to complement set.

Step 3. Calculate the weighted z-score function for HFS as in Definition 3.13-Definition 3.15.

Step 4. Determine the ranking for the alternatives involved.

Step 5. Choose the best alternative.

Example 3.6. [33] To describe the proposed results, examples adapted from [34] are used. This example assumes that an automotive company seeks to choose a supplier that is best suited for one of the key elements of its manufacturing process. After the initial assessment, four suppliers are shortlisted for further assessment. For more accurate assessments on different suppliers, four of the most important criteria have been selected as follows $E_{1}$ : product quality; $E_{2}$ : relationship closeness; $E_{3}$ : delivery reputation; and $E_{4}$ : price. Clearly, $E_{1}, E_{2}$ and $E_{3}$ are benefit criteria, and $E_{4}$ is cost criterion. To comprehensively evaluate various suppliers, experts who have different skills, experience and knowledge values were invited to conduct an assessment. The criteria weight vector is $w=(0.2249,0.2357,0.2560,0.2833)$ [35]. The information provided by the experts is presented in the form of HFE matrix as provided in Table 8 .

Table 8. Decision matrix of HFS

\begin{tabular}{|c|c|c|c|c|}
\hline & $E_{1}$ & $E_{2}$ & $E_{3}$ & $E_{4}$ \\
\hline$S_{1}$ & $\{0.2,0.4,0.7\}$ & $\{0.1,0.2,0.5,0.7\}$ & $\{0.2,0.3,0.5,0.7,0.8\}$ & $\{0.1,0.4,0.6\}$ \\
\hline$S_{2}$ & $\{0.4,0.6,0.7\}$ & $\{0.1,0.2,0.4,0.6\}$ & $\{0.3,0.4,0.6,0.8,0.9\}$ & $\{0.1,0.2,0.4\}$ \\
\hline$S_{3}$ & $\{0.2,0.3,0.6\}$ & $\{0.3,0.4,0.5,0.9\}$ & $\{0.2,0.4,0.6,0.7,0.8\}$ & $\{0.3,0.4,0.8\}$ \\
\hline$S_{4}$ & $\{0.2,0.3,0.5\}$ & $\{0.2,0.3,0.5,0.7\}$ & $\{0.4,0.6,0.7,0.8,0.9\}$ & $\{0.1,0.2,0.7\}$ \\
\hline
\end{tabular}

Based on the proposed algorithm, Table 9 to Table 13 show z-score and ranking values for different $(\alpha, \beta)$.

Table 9. Score and ranking with $(\alpha, \beta)=(1,1)$

\begin{tabular}{|c|c|c|c|c|c|}
\hline Algorithm & $S_{1}$ & $S_{2}$ & $S_{3}$ & $S_{4}$ & Ranking \\
\hline z-arithmetic mean & 0.7089 & 0.8800 & 0.7167 & 0.7774 & $S_{1} \prec S_{3} \prec S_{4} \prec S_{2}$ \\
\hline z-geometric mean & 0.6663 & 0.8530 & 0.6835 & 0.8039 & $S_{1} \prec S_{3} \prec S_{4} \prec S_{2}$ \\
\hline z-harmonic mean & 0.6412 & 0.8247 & 0.6482 & 0.8230 & $S_{1} \prec S_{3} \prec S_{4} \prec S_{2}$ \\
\hline
\end{tabular}

Table 10. Score and ranking with $(\alpha, \beta)=(0.5,0.5)$

\begin{tabular}{|c|c|c|c|c|c|}
\hline Algorithm & $S_{1}$ & $S_{2}$ & $S_{3}$ & $S_{4}$ & Ranking \\
\hline z-arithmetic mean & 0.7085 & 0.8833 & 0.7159 & 0.7995 & $S_{1} \prec S_{3} \prec S_{4} \prec S_{2}$ \\
\hline z-geometric mean & 0.6616 & 0.8483 & 0.6814 & 0.8311 & $S_{1} \prec S_{3} \prec S_{4} \prec S_{2}$ \\
\hline z-harmonic mean & 0.6300 & 0.8063 & 0.6390 & 0.8437 & $S_{1} \prec S_{3} \prec S_{4} \prec S_{2}$ \\
\hline
\end{tabular}


Table 11. Score and ranking with $(\alpha, \beta)=(1,1)$

\begin{tabular}{|c|c|c|c|c|c|}
\hline Algorithm & $S_{1}$ & $S_{2}$ & $S_{3}$ & $S_{4}$ & Ranking \\
\hline z-arithmetic mean & 0.7090 & 0.8867 & 0.7156 & 0.8233 & $S_{1} \prec S_{3} \prec S_{4} \prec S_{2}$ \\
\hline z-geometric mean & 0.6578 & 0.8434 & 0.6798 & 0.8603 & $S_{1} \prec S_{3} \prec S_{2} \prec S_{4}$ \\
\hline z-harmonic mean & 0.6050 & 0.8063 & 0.6231 & 0.8471 & $S_{1} \prec S_{3} \prec S_{2} \prec S_{4}$ \\
\hline
\end{tabular}

Table 12. The score and ranking with $(\alpha, \beta)=(0,1)$

\begin{tabular}{|c|c|c|c|c|c|}
\hline Algorithm & $S_{1}$ & $S_{2}$ & $S_{3}$ & $S_{4}$ & Ranking \\
\hline z-arithmetic mean & 0.7090 & 0.8867 & 0.7156 & 0.8233 & $S_{1} \prec S_{3} \prec S_{4} \prec S_{2}$ \\
\hline z-geometric mean & 0.6578 & 0.8434 & 0.6798 & 0.8603 & $S_{1} \prec S_{3} \prec S_{2} \prec S_{4}$ \\
\hline z-harmonic mean & 0.6050 & 0.7727 & 0.6231 & 0.8471 & $S_{1} \prec S_{3} \prec S_{2} \prec S_{4}$ \\
\hline
\end{tabular}

Table 13. Score and ranking with $(\alpha, \beta)=(1,0)$

\begin{tabular}{|c|c|c|c|c|c|}
\hline Algorithm & $S_{1}$ & $S_{2}$ & $S_{3}$ & $S_{4}$ & Ranking \\
\hline z-arithmetic mean & 0.7086 & 0.8800 & 0.7167 & 0.7774 & $S_{1} \prec S_{3} \prec S_{4} \prec S_{2}$ \\
\hline z-geometric mean & 0.6663 & 0.8530 & 0.6835 & 0.8039 & $S_{1} \prec S_{3} \prec S_{4} \prec S_{2}$ \\
\hline z-harmonic mean & 0.6412 & 0.8247 & 0.6482 & 0.8230 & $S_{1} \prec S_{3} \prec S_{4} \prec S_{2}$ \\
\hline
\end{tabular}

Table 9 - Table 13 show the alternative ranking when parameters are evaluated differently. Decision makers with different subjective options can choose certain parameters according to their experience and attitude. This means that the proposed z-score is beneficial for a combination of subjective and objective decision-making information.

Sensitivity analysis is done by modifying parameters $(\alpha, \beta)$ (level of interest for the hesitant degree and deviation degree) by increasing and decreasing the parameters. The ranking for alternatives is the same as obtained by Xu and Xia [33] and Hu et.al [35].

\section{Conclusions}

Some of the conclusions of this study are as follows:

1. The novel hesitant degree of HFE can escape the propensity of decision makers to evaluate an entity or criteria by restricting hesitancy.

2. The arithmetic mean, geometric mean and harmonic mean score functions can be integrated with the hesitant degree of HFE and deviation of HFE and are used to solve some of the challenges encountered by the current score function to make it more flexible. Decision makers set certain parameters based on their expertise or knowledge to measure the z-score functions.

3. By utilizing the recommended z-score function, the MCDM solution process can be achieved. The provided algorithm is simpler and compatible with current algorithms.

4. The proposed algorithm may prevent MCDM from applying any membership to a shorter HFE and render it equivalent to another HFE, or repeating their membership to achieve two series of the same length, thus losing the original data structure and modifying the HFE data details [36].

Future experiments might be extended to different fields for the data recruitment process and different algorithms such as Technique for Order of Preference by Similarity to Ideal Solution (TOPSIS), VlseKriterijumska Optimizcija I Kaompromisno Resenje (VIKOR), The Preference Ranking Organization METHod for Enrichment of Evaluations (PROMETHEE) and ELimination Et Choix Traduisant la REalité (ELECTRE) in MCDM.

\section{REFERENCES}

[1] L. . Zadeh, "Fuzzy Sets,” Inf. Control, vol. 8, pp. 338-353, 1965 , https://doi.org/10.1016/S0019-9958(65)90241-X.

[2] K. T. Atanassov, "INTUITIONISTIC FUZZY SETS," Fuzzy Sets Syst., vol. 20, pp. 87-96, 1986.

[3] K. Atanassov and G. Gargov, "interval-valued intuitionistic fuzzy sets,” Fuzzy Sets Syst., vol. 31, no. 3, pp. 343-349, 1989, doi: 10.1016/S0165-0114(98)00436-9.

[4] R. R. Yager, “On the theory of bags,” Int. J. Gen. Syst., vol. 13, no. 1, pp. 23-37, 1986, doi: $10.1080 / 03081078608934952$.

[5] P. K. Maji, R. Biswas, and A. R. Roy, "Soft set theory," J. Fuzzy Math., vol. 9, no. 3, pp. 589-602, 2003, doi: 10.1016/S0898-1221(03)00016-6. 
[6] V. Torra, “Hesitant Fuzzy Sets,” Int. J. Intell. Syst., vol. 25, pp. 529-539, 2010, doi: 10.1002/int.

[7] V. Torra and Y. Narukawa, "On hesitant fuzzy sets and decision,” IEEE Int. Conf. Fuzzy Syst., pp. 1378-1382, 2009, doi: 10.1109/FUZZY.2009.5276884.

[8] H. Zhang and L. Shu, "dual hesitant fuzzy soft set and its properties,” Adv. Intell. Syst. Comput., vol. 367, pp. 171182, 2016, doi: 10.1007/978-3-319-19105-8.

[9] J. Chen and X. Huang, "Dual hesitant fuzzy probability," Symmetry (Basel)., vol. 9, no. 4, 2017, doi: 10.3390/sym9040052.

[10] José Carlos R. Alcantud and J. Z. Gustavo Santos-García, Xindong Peng, "Dual Extended Hesitant Fuzzy Sets," Symmetry (Basel)., vol. 11, no. 714, pp. 1-13, 2019, doi: 10.3390/sym 11050714 .

[11] Z. Rodzi and A. G. Ahmad, "Fuzzy Parameterized Hesitant Fuzzy Linguistic Term Soft Sets (FPHFLTSSs) in Multi-Criteria Decision Making," Int. J. Innov. Technol. Explor. Eng., vol. 9, no. 5, pp. 909-916, 2020, doi: 10.35940/ijitee.E2519.039520.

[12] Z. Rodzi and A. G. Ahmad, "Application of Parameterized Hesitant Fuzzy Soft Set Theory in Decision Making,” Math. Stat., vol. 8, no. 3, pp. 244-253, 2020, doi: 10.13189/ms.2020.080302.

[13] Z. Rodzi and A. G. Ahmad, "Fuzzy Parameterized Dual Hesitant Fuzzy Soft Sets and Its Application in TOPSIS," Math. Stat. 8, vol. 8, no. 1, pp. 32-41, 2020, doi: 10.13189/ms.2020.080104.

[14] B. Zhu, Z. Xu, and M. Xia, "Dual hesitant fuzzy sets,” J. Appl. Math., vol. 2012, 2012, doi: 10.1155/2012/879629.

[15] D. K. Joshi, I. Beg, and S. Kumar, "Hesitant probabilistic fuzzy linguistic sets with applications in multi-criteria group decision making problems," Mathematics, vol. 6, no. 4, 2018, doi: 10.3390/math6040047.

[16] H. Liao, Z. Xu, X. J. Zeng, and J. M. Merigó, “Qualitative decision making with correlation coefficients of hesitant fuzzy linguistic term sets,” Knowledge-Based Syst., vol. 76, no. December, pp. 127-138, 2015, doi: 10.1016/j.knosys.2014.12.009.

[17] R. M. Rodriguez, L. Martinez, and F. Herrera, "Hesitant fuzzy linguistic term sets for decision making," IEEE Trans. Fuzzy Syst., vol. 20, no. 1, pp. 109-119, 2012, doi: 10.1109/TFUZZ.2011.2170076.

[18] A. Mardani, M. K. Saraji, A. R. Mishra, and P. Rani, “A novel extended approach under hesitant fuzzy sets to design a framework for assessing the key challenges of digital health interventions adoption during the COVID-19 outbreak,” Appl. Soft Comput. J., vol. 96, p. 106613, 2020, doi: 10.1016/j.asoc.2020.106613.

[19] J. ying Dong, F. fang Yuan, and S. ping Wan, "Extended VIKOR method for multiple criteria decision-making with linguistic hesitant fuzzy information," Comput. Ind. Eng., vol. 112, pp. 305-319, 2017, doi: 10.1016/j.cie.2017.07.025.

[20] G. Sun, X. Guan, X. Yi, and Z. Zhou, “An innovative TOPSIS approach based on hesitant fuzzy correlation coefficient and its applications,” Appl. Soft Comput. J., vol.
68, pp. 249-267, 2018, doi: 10.1016/j.asoc.2018.04.004.

[21] N. Ghorui et al., "Identification of dominant risk factor involved in spread of COVID-19 using hesitant fuzzy MCDM methodology,” Results Phys., vol. 21, p. 103811, 2021, doi: 10.1016/j.rinp.2020.103811.

[22] R. Wang and Y. Li, "Generalized single-valued neutrosophic hesitant fuzzy prioritized aggregation operators and their applications to multiple criteria decision-making," Inf., vol. 9, no. 1, 2018, doi: 10.3390/info9010010.

[23] Z. Bashir, Y. Bashir, T. Rashid, J. Ali, and W. Gao, “A Novel Multi-Attribute Group Decision-Making Approach in the Framework of Proportional Dual Hesitant Fuzzy Sets," Appl. Sci., vol. 9, no. 6, p. 1232, 2019, doi: 10.3390/app9061232.

[24] N. Lu and L. Liang, "Correlation coefficients of extended hesitant fuzzy sets and their applications to decision making," Symmetry (Basel)., vol. 9, no. 4, 2017, doi: 10.3390/sym9040047.

[25] B. Wang, J. Liang, and J. Pang, "Deviation Degree: A Perspective on Score Functions in Hesitant Fuzzy Sets," Int. J. Fuzzy Syst., vol. 21, no. 7, pp. 2299-2317, 2019, doi: 10.1007/s40815-019-00722-x.

[26] M. Xia and Z. Xu, "Hesitant fuzzy information aggregation in decision making,” Int. J. Approx. Reason., vol. 52, no. 3, pp. 395-407, 2011, doi: 10.1016/j.ijar.2010.09.002.

[27] H. Liao and Z. Xu, "A VIKOR-based method for hesitant fuzzy multi-criteria decision making,” Fuzzy Optim. Decis. Mak., vol. 12, no. 4, pp. 373-392, 2013, doi: 10.1007/s10700-013-9162-0.

[28] X. Zhang and Z. Xu, “The TODIM analysis approach based on novel measured functions under hesitant fuzzy environment," Knowledge-Based Syst., vol. 61, pp. 48-58, 2014, doi: 10.1016/j.knosys.2014.02.006.

[29] B. Farhadinia, "Information measures for hesitant fuzzy sets and interval-valued hesitant fuzzy sets," Inf. Sci. ( Ny)., vol. 240, pp. 129-144, 2013, doi 10.1016/j.ins.2013.03.034.

[30] B. Farhadinia, "A series of score functions for hesitant fuzzy sets,” Inf. Sci. (Ny)., vol. 277, pp. 102-110, 2014, doi: 10.1016/j.ins.2014.02.009.

[31] D. Li, W. Zeng, and J. Li, "New distance and similarity measures on hesitant fuzzy sets and their applications in multiple criteria decision making,” Eng. Appl. Artif. Intell., vol. 40, pp. 11-16, 2015, doi: 10.1016/j.engappai.2014.12.012.

[32] N. Chen, Z. Xu, and M. Xia, "The ELECTRE I multi-criteria decision-making method based on hesitant fuzzy sets,” Int. J. Inf. Technol. Decis. Mak., vol. 14, no. 3, pp. 621-657, 2015, doi: 10.1142/S0219622014500187.

[33] Z. Xu and Meimei Xia, "Hesitant Fuzzy Entropy and Cross-Entropy and Their Use in Multiattribute Decision-Making,” Int. J. Intell. Syst., vol. 27, pp. 799-822, 2012, doi: 10.1002/int.

[34] F. E. Boran, S. Genç, M. Kurt, and D. Akay, “A multi-criteria intuitionistic fuzzy group decision making for supplier selection with TOPSIS method,” Expert Syst. Appl., 
vol. 36, no. 8, pp. 11363-11368, 2009, doi: 10.1016/j.eswa.2009.03.039.

[35] J. Hu, X. Zhang, X. Chen, and Y. Liu, "Hesitant fuzzy information measures and their applications in multi-criteria decision making,” Int. J. Syst. Sci., vol. 47, no.
1, pp. 62-76, 2016, doi: 10.1080/00207721.2015.1036476.

[36] J. H. Lv, S. C. Guo, and F. F. Guo, "Study on Hesitant Fuzzy Information Measures and Their Clustering Application,” Comput. Intell. Neurosci., vol. 2019, 2019, doi: 10.1155/2019/5370763. 